\title{
Test Preparation Asparagus Total Polysaccharides of Scanning and Standard Curve
}

\author{
Yong-Guang $\mathrm{BI}^{1,}{ }^{,}$, Hai-Lan HUANG ${ }^{1}$, Zhong WANG ${ }^{2}$,Xue-Wei \\ $\mathrm{CHEN}^{1}$,Xue-Mei LIU ${ }^{1}$, Shi-Ting DENG ${ }^{1}$ and Hong $\mathrm{YU}^{1}$ \\ ${ }^{1}$ School of Pharmacy, Guangdong Pharmaceutical University, Guangzhou 510006, Guangdong, \\ China \\ ${ }^{2}$ School of Clinical Medicine, Guangdong Pharmaceutical University, Guangzhou 510006, \\ Guangdong, China \\ E-mail:biyongguang2002@163.com
}

Keywords:Asparagus dishes, Total polysaccharide,Standard curve,Wavelength scan

\begin{abstract}
This paper discusses the peak of both glucose uptake and standards of polysaccharides are Gracilaria maximum absorption at $485 \mathrm{~nm}$, it is OK to select UV measured drawing and polysaccharide content in the sample solution of polysaccharide from Gracilaria glucose standard curve absorptiometry a wavelength of $485 \mathrm{~nm}$. The results show that: the regression equation of the standard curves for glucose, correlation coefficient, indicating that the glucose concentration in the standard range $(0 \sim 0.1 \mathrm{mg})$ with absorbance good linear relationship.
\end{abstract}

\section{Introduction}

Life comes from the sea, about $70 \%$ of the Earth's surface is covered by oceans, and our country is coastal country with a long coastline, in recent years, the development and use of living marine scientific research is the hot topic, we all know, a wide variety of marine life, with a wide variety of biological activity and development value. Seaweed is an important part of marine life, with the rapid development of China's marine drug research, particularly the study of marine algae is more extensive and in-depth [1]. Which is widely human development and utilization of large-scale algae mainly Rhodophyta (such as seaweed, agar, etc.), Phaeophyceae (such as kelp, horsetail, etc.) and green algae (Ulva, Codium, etc.) and other three class. Marine life has its special chemical structure and biological activity and special features, its diversity, complexity and particularity of human looking marine bioactive substances provides a rich source. Polysaccharides (of polysaccharide) is a sugar chain by a glycosidic bond is formed, at least to more than 10 or more monosaccharide polymeric sugar carbohydrate polymer, represented by the formula $\left(\mathrm{C}_{6} \mathrm{H}_{10} \mathrm{O}_{5}\right) \mathrm{n}$ represents. As well as storing nutrients and backbone component of the plant, many studies and reports suggest that polysaccharides have extensive and good biological activity, such as anti-tumor and immune boosting, anti-inflammatory, anti-viral, anti-coagulation, anti-aging activity. Polysaccharide with increasing degree of polymerization, generally amorphous, insoluble in water, soluble in hot water or a colloidal solution, no sweetness, but in nature, most of the polysaccharide to form a glycoside, glycoside soluble in water, so water can be the most solvent extraction, economical and environmentally friendly.

Rich in marine resources, there is about 200,000 kinds of marine life, including a wide variety of marine plants, accounting for about one-eighth of marine life. Rich marine plant resources are usedin medicine, health, food, textile and other fields of development and their utilization increasingly widespread. Since 1881, the British Stanford discovered algin from brown algae, seaweed polysaccharides industrial production began to develop rapidly. Seaweed polysaccharide because of its unique biological activity, natural pollution-free, widely available, has great potential in the development of health supplies, so widespread concern in the scientific community.

Numerous literatures show that many seaweed monosaccharides with some special group or a substituent group such as sulfuric acid, pyruvic acid, etc. on the composition, which determines its particularity. But generally it has water-soluble polysaccharide seaweed, mostly containing sulfuric 
acid group, mostly solidified strong or high viscosity, so that it is limited in clinical application. To solve the molecular polysaccharide polymer material is too large, high viscosity, poor solubility, and can be considered degradable polymer seaweed polysaccharides, oligosaccharides obtained polysaccharide fragments or small molecules to enhance their body through the barrier of flexibility, so as to more fully achieve biological activity. From the following points to consider: First, the glycan structures are single or two or cross monosaccharide composition, and without too many branches, so to obtain various degrees of polymerization of sugar, secure the reproducibility will be more activity for sex. Secondly, oligosaccharides also in the process of cellular activities important coordinating substance identification adhesion, cell recognition between viral and bacterial intercellular require the participation of oligosaccharides. Therefore, it is considered by chemical modification, change polysaccharide complex structure to expose more active groups, making it play a more prominent new property. And in recent years, contact between the structure and function of oligosaccharides gradually become an issue of interest to the academic community, hoping to provide a basis for the study of new drugs or ocean marine health products.

Seaweed belongs to a class of polysaccharides seaweed extract, since they have good viscosity and chemical properties coagulation, emulsification and film, etc., and therefore has a wide range of value, such as agar, carrageenan, alginate, etc. It has long been mass produced and used in industry, but also as a coagulant, a thickener, an excipient, emulsifier and dispersing agent widely used in pharmaceutical, chemical and food industries [2]. With seaweed polysaccharide more in-depth study and understanding, seaweed polysaccharides as potential function of drugs and drug intermediates increasingly recognized and utilized, seaweed polysaccharides exhibit anticoagulant activity, anti-tumor and anti-viral activity increases the breadth of its medicinal value. Seaweed polysaccharides can be used as bio-absorber and nutrient resources, protection of cellular material cold agent, lowering blood pressure, anti-oxidation, anti-tumor, enhance immune modulating activity, including red algae with Gracilaria represented Chen Meizhen other studies have shown: Long shall be food polysaccharide could significantly inhibit the immunosuppressant cyclophosphamide peptide amine (CP) induced micronucleus and sperm abnormality, and there is a certain dose-effect relationship.

Gracilaria (Gracilaria verrucosa), also known as Gracilaria, carrageenan, seaweed and other Department of Rhodophyta, Gracilariaceae Gracilaria genus Gracilaria (Graciria lemaneiformis) of algae, originating in China, Qinghai, Shandong Province and Okinawa, Japan. In recent years, with the upgrading and development of cultivation techniques through artificial breeding, asparagus has been successful introduction from Qingdao to Fujian, Guangdong, Zhejiang and other coastal areas, and gradually developed into the area's main economic algae with a scale, with high economic and ecological value. Asparagus dish is rich in protein, fat, dietary fiber, trace elements and vitamins and other ingredients, it is a high-quality natural health food ingredients. Studies have shown that various types of ingredients contained in Gracilaria have good pharmacological activities: Polysaccharides having anti-tumor [, anti-viral effects, phycobiliprotein, an organic acid also has anti-tumor effect, dietary fiber has obvious Run intestinal catharsis [3]. It became clear that the asparagus dish has not only limited for extracting agar and as feed, there is a big space for development and use of its main active ingredient, has a considerable commercial value and medicinal value, such as polysaccharides and proteins seaweed has been widely used in medicine, food, cosmetics and other fields, so for asparagus dishes active ingredients strengthen the deepening of research, and constantly improve the extraction process of the active ingredients, reduce economic costs, streamline operations, and easy way to find a more efficient process, make it play a broader role and asparagus dish as a whole reflects the economic value of large seaweed resources is particularly important and significant in the fields of medicine, food and health care.

Polysaccharide from Gracilaria general extraction methods include cold water extraction, hot water extraction, enzymatic extraction method, microwave and ultrasonic assisted extraction, etc.[4], it has been reported. Advantages With the continuous development of science and technology and improved ultrasonic extraction technology is now widely used in the extraction process plant polysaccharides, and continue research suggests that ultrasound-assisted extraction technique has 
generally improved extraction efficiency, accelerate the dissolution of the active ingredient, easy operation,etc..Ultrasonic extraction method is the application of ultrasound enhancement technology to extract active ingredients of plant, is a physical crushing process , mechanical grinding mechanism is mainly the use of ultrasonic, thermal mechanism and cavitation damage to the cell wall of natural medicines, the active ingredient dissolution, so as to achieve the purpose of improving the extraction rate of the active ingredient. The media have a special ultrasonic mechanical vibration and cavitation, can produce and deliver powerful energy when the ultrasonic vibration applied to the liquid, when the liquid is sparse state, the liquid will be torn into tiny holes generated when the hole is closed high-voltage transient, called cavitation. Cavitation can refine material damage cell wall structure so as to form an emulsion state, accelerating the active ingredient into the solvent to improve the dissolution of the active substance.

This experiment focuses on the preparation of the maximum absorption wavelength range of the standard curve and asparagus dish sample solution, in order to lay a solid foundation for future research.

\section{The Experimental Materials and Instrument}

Stem asparagus, place of origin: Shandong.Swing-speed grinder: Wenling Lin Machinery Co.; AY120 electronic analytical balance: Shimadzu Corporation; KH-400KDB High Power CNC ultrasonic cleaner: Ultrasonic Instrument Co., Ltd. Kunshan Wo Chong; SHZ-D (III) circulating water pumps: Gongyi City to Hua instrument Co., Ltd.; RE-52AA rotary evaporator: Haiya Rong Biochemical instrument Factory.

\section{Experimental Methods}

\section{Sample Preparation}

The asparagus dish (dried) after pulverizer, over 40 mesh sieve, sealed bottles back.

\section{Preparation of the Glucose Reference Solution}

Precision Weigh glucose reference substance $20.0 \mathrm{mg}$, set $100 \mathrm{~mL}$ flask, add distilled water and diluted to volume. Shake, that glucose stock solution (1mL each containing glucose 200ug).

\section{Glucose Absorption Wavelength Selection}

Determination of polysaccharide content asparagus dishes are mainly two, namely anthrone sulfuric acid and phenol - sulfuric acid method. Yang Xianqing other study found that phenol sulfuric acid method is more reasonable and accurate and sensitive than anthrone - sulfuric acid method, the asparagus dishes determination of polysaccharide may give priority to the phenol sulfuric acid method, phenol - sulfuric acid method is more simple and sensitive method basically not affected by the experiment measuring the protein, and measurement results are relatively stable in 60min, so the present study phenol - sulfuric acid method, and reference spectral scanning methods and standard curve of glucose absorption wavelength pretreatment drawn jobs.

\section{Glucose Standard Curve Draw}

The precise amount of glucose stock solution (200ug/mL) in 10 $0.05,0.10,0.15,0.20,0.25,0.30,0.35,0.40,0.45,0.50 \mathrm{~mL}$ each test tube with distilled water up to $1 \mathrm{~mL}$, then were added $6 \%$ phenol $1 \mathrm{~mL}$, shake, and then quickly added concentrated sulfuric acid $5 \mathrm{~mL}$, shake well and let cool to room temperature, $40{ }^{\circ} \mathrm{C}$ water bath $20 \mathrm{~min}$, let cool to room temperature; another $1 \mathrm{~mL}$ with distilled water as a control treatment, and to measure the absorbance at $485 \mathrm{~nm}$. Absorbance A for the vertical axis, glucose C (ug) as the abscissa, the standard curve (Figure 2).

\section{Results and Discussion}

Depicting the right amount of glucose standard and Gracilaria polysaccharide extract with treatment, 
then spectral scanning, spectrums scanning have both shown in Fig.1:

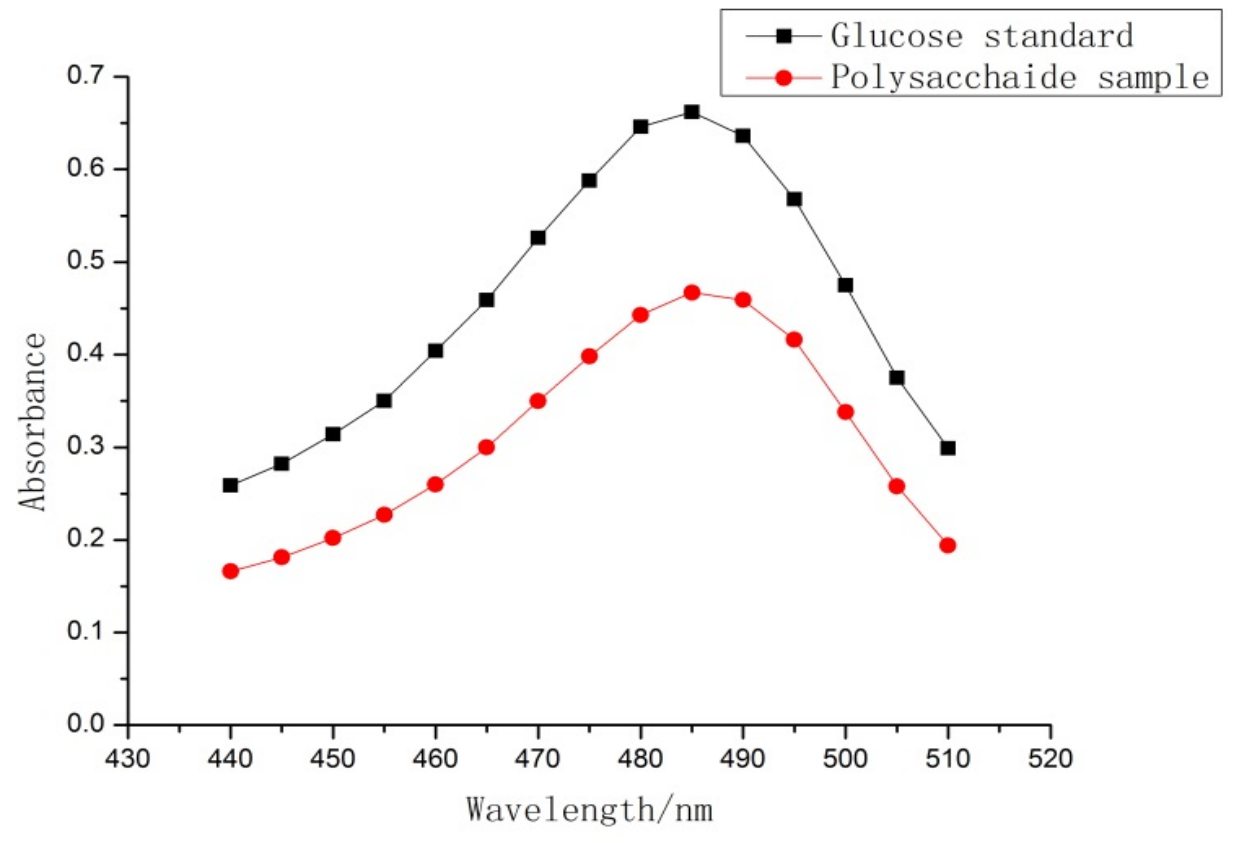

Fig.1 Standard and Gracilaria glucose polysaccharide extract UV - visible spectrum scan comparison chart

According to Fig. 1 shows that both are at $485 \mathrm{~nm}$ of maximum absorption, it is determined to select a wavelength of 485nm UV absorptiometry measured drawing and polysaccharide content in the sample solution of polysaccharide from Gracilaria glucose standard curve.

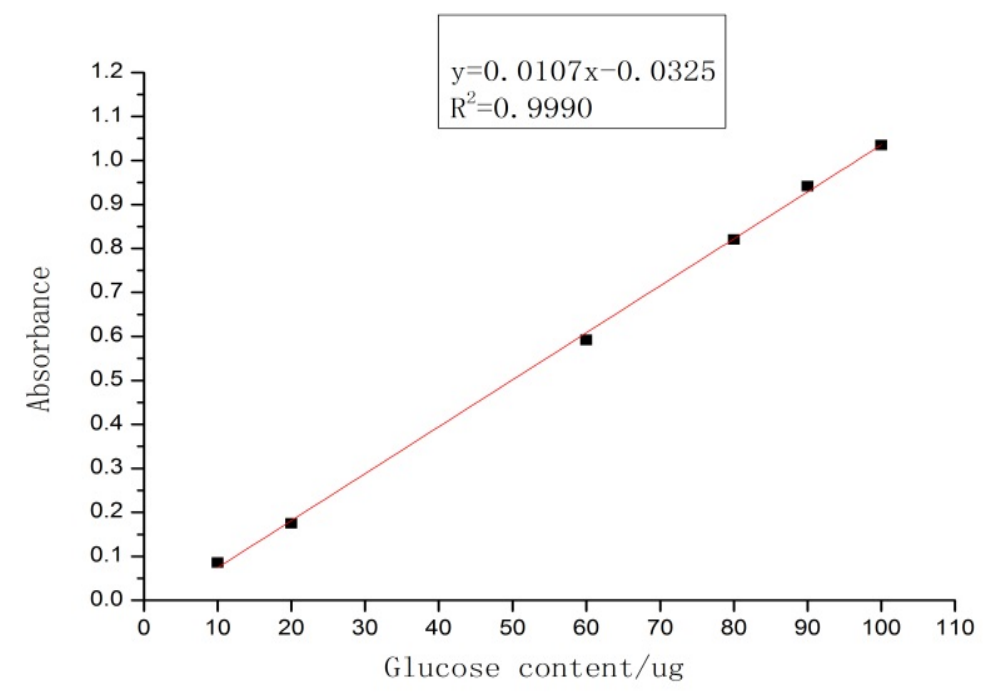

Fig.2 Glucose standard curve

Fig. 2 shows the regression equation for glucose standard curve, correlation coefficient, indicating that the glucose concentration in the standard range $(0 \sim 0.1 \mathrm{mg})$ with absorbance good linear relationship.

\section{Conclusion}

(1)This paper tests finalize select UV absorption measurement wavelength of $485 \mathrm{~nm}$ at 
Determination of Polysaccharide from Gracilaria polysaccharide sample solution by. The results show that: the regression equation of the standard curves for glucose, correlation coefficient, indicating that the glucose concentration in the standard range $(0 \sim 0.1 \mathrm{mg})$ with absorbance good linear relationship.(2)In this paper, the use of ultraviolet-visible spectrophotometer scanning, sample drawings and standards consistent with maximum absorption peak, indicating that the method chosen scientific paper, reasonable and practicable, which has a reference value.

\section{Acknowledgements}

This work is supported by Guangdong Provincial Oceanic and Fishery Bureau - marine fisheries science and technology and industrial development projects (No. A201501C11).

\section{References:}

[1]Dhargalkar VK, Verlecar XN.Southern Ocean seaweeds:A resource for exploration in food and drugs[J].Aquaclture, 2009,287(3-4):229-242.

[2]Siddhanta AK.Marine algal polysaccharides-functions and utilization Trends Carbohydr Chen[C].Carbohydr Conf $9^{\text {th }} 1993$, India:Surya International Publications, 1995:125-131.

[3]Souza B W,Cerqueira M A, Bourbon A I,et al.Chemical characterization and antioxidant activity of sulfated polysaccharide from the red seaweed Gracilaria birdiae[J]. Food Hydrocolloids, 2012,27(2):287-292.

[4]Smironff N,Cumbes Q J. Hyroxyl radical scavenging activity of compatible solutes[J]. Phytochemistry, 1989, 28:1057-1060. 